TITLE:

\title{
Nonlinear parity mixtures controlling the propagation of interchange modes
}

\author{
$\operatorname{AUTHOR}(\mathrm{S})$ :
}

Sato, M.; Ishizawa, A.

\section{CITATION:}

Sato, M....[et al]. Nonlinear parity mixtures controlling the propagation of interchange modes. Physics of Plasmas 2017, 24(8): 082501.

\section{ISSUE DATE:}

2017-07

URL:

http://hdl.handle.net/2433/261132

\section{RIGHT:}

This article may be downloaded for personal use only. Any other use requires prior permission of the author and AIP Publishing. This article appeared in Physics of Plasmas 24, 082501 (2017) and may be found at https://doi.org/10.1063/1.4993472. 


\section{Nonlinear parity mixtures controlling the propagation of interchange modes}

Cite as: Phys. Plasmas 24, 082501 (2017); https://doi.org/10.1063/1.4993472

Submitted: 20 April 2017 . Accepted: 28 June 2017 . Published Online: 18 July 2017

M. Sato, and A. Ishizawa

\section{ARTICLES YOU MAY BE INTERESTED IN}

Comprehensive magnetohydrodynamic hybrid simulations of fast ion driven instabilities in a Large Helical Device experiment

Physics of Plasmas 24, 081203 (2017); https://doi.org/10.1063/1.4997529

Modeling of turbulent particle and heat transport in helical plasmas based on gyrokinetic analysis

Physics of Plasmas 26, 012510 (2019); https://doi.org/10.1063/1.5058720

Excitation of macromagnetohydrodynamic mode due to multiscale interaction in a quasisteady equilibrium formed by a balance between microturbulence and zonal flow Physics of Plasmas 14, 040702 (2007); https://doi.org/10.1063/1.2716669

\section{AIP Advances Fluids and Plasmas Collection}

\section{READ NOW}

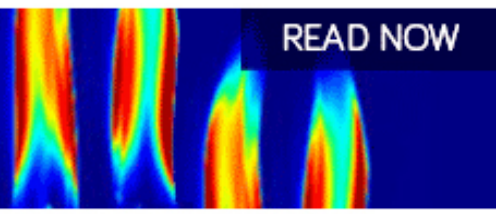




\title{
Nonlinear parity mixtures controlling the propagation of interchange modes
}

\author{
M. Sato ${ }^{1}$ and A. Ishizawa ${ }^{2}$ \\ ${ }^{1}$ National Institute for Fusion Science, National Institutes of Natural Sciences, Toki 509-5292, Japan \\ ${ }^{2}$ Graduate School of Energy Science, Kyoto University, Uji, Kyoto 611-0011, Japan
}

(Received 20 April 2017; accepted 28 June 2017; published online 18 July 2017)

\begin{abstract}
The propagation velocity of a resistive interchange mode is numerically investigated based on a two-fluid model. It is newly found that the nonlinearity mixes the interchange parity and the tearing parity to produce magnetic islands and controls the propagation velocity of the instability in the poloidal direction. The parity of the interchange mode is conserved during the linear growing evolution. However, when the amplitude of the mode becomes large and nonlinear effects are dominant, the pure interchange mode does not satisfy the nonlinear two-fluid equation. Thus, the nonlinear energy transfer occurs from the interchange parity mode to the tearing parity mode, which is called the nonlinear parity mixtures, and the magnetic islands are produced by the interchange mode. The nonlinear magnetic island formation by the interchange mode plays a central role in controlling the interchange mode's propagation velocity, which is equal to the electron fluid velocity. This nonlinear process is essential in quantitatively reproducing the propagation velocity of the interchange mode, which is the same as the electron fluid velocity observed in the large helical device experiment. It is also found that one of the mechanisms of parity mixtures is a modulational instability. Published by AIP Publishing. [http://dx.doi.org/10.1063/1.4993472]
\end{abstract}

\section{INTRODUCTION}

One of the critical issues in the confinement of helical plasmas is to suppress the pressure driven magnetohydrodynamic (MHD) instabilities. Experimental results from the large helical device (LHD) suggest that the mode rotation plays an important role in the MHD activity. For instance, a locked mode-like instability was found when the magnetic shear is weak in the entire plasma region in LHD experiments. ${ }^{1,2}$ In the locked mode-like instability, a rotating resistive interchange mode is often observed as a precursor. When the rotation of the resistive interchange mode stops, the amplitude of the mode rapidly increases and then a minor collapse occurs. In addition, when resistive interchange modes rotate in the poloidal direction in the observation of high beta LHD plasmas with high magnetic shear in the peripheral region, the influence of the instabilities on the plasma confinement is small. ${ }^{3}$ Recently, it is found that the rotation velocity of the magnetic perturbation of the interchange mode agrees well with the electron fluid velocity in the LHD plasmas. ${ }^{4}$ The rotation velocity has been studied by numerical simulations based on fluid models. However, the linear analysis of the interchange mode ${ }^{5}$ is not in agreement with the rotation velocity observed in LHD experiments. The linear analysis based on the two-fluid reduced model has been also carried out by focusing on the influence of plasma viscosity and thermal conductivity. ${ }^{6}$ The present study has been undertaken to examine the influence of the nonlinear formation of the magnetic islands on the propagation velocity of the resistive interchange mode. The study is motivated by finding that magnetic islands are nonlinearly formed in the previous nonlinear simulation studies based on the threefield model without the two-fluid effects, ${ }^{7,8}$ although the interchange mode does not generate the magnetic islands in the linear phase. The formation of the magnetic islands may affect the rotation velocity of the modes, ${ }^{9}$ and it is linked to the parity of the interchange mode. ${ }^{10}$ The interchange mode has a specific dependence on the coordinate in the radial direction, and its electrostatic potential is an even function of the local radial coordinate around the mode resonance surface. This is clearly represented by the equation $\phi(-x)$ $=\phi(x)$, where $x=r-r_{s}$, and the mode having this parity is called as the interchange parity mode. On the other hand, when electrostatic potential is an odd function, $\phi(-x)=-\phi(x)$, the mode is called as the tearing parity mode. In the linear growing evolution, these parities are independent. However, they are mixed in the nonlinear evolution, and this mixture has a significant impact on the saturation level of instability, especially on the formation of magnetic islands. ${ }^{10}$ This paper presents the dependence of the propagation velocity of the interchange mode on the width of the magnetic islands produced by nonlinear parity mixtures by means of the twofluid simulations. Since the formation of the magnetic islands is represented by the appearance of the tearing parity mode, the generation of the tearing parity mode is also discussed in detail. It is found that a modulational instability is a typical process of the nonlinear tearing parity mode production.

The remainder of this paper is as follows: The numerical model is shown in Sec. II. The parity of instabilities is discussed in Sec. III. The linear analysis of the interchange mode is presented in Sec. IV, and the nonlinear evolution obtained from the simulations is presented in Sec. V. Finally, summary is given in Sec. VI.

\section{NUMERICAL MODEL}

In this paper, we use the four-field model, which is a typical two-fluid model for the analysis of instabilities caused in magnetized plasmas, ${ }^{11}$ and we consider, for simplicity, two dimensional slab geometry by assuming that the 
plasma is uniform along the $z$-axis. The four-field model consists of the vorticity equation, the generalized Ohm's law, the pressure equation, and the parallel velocity equation

$$
\begin{gathered}
\frac{\partial U}{\partial t}=[U, \phi]+[\psi, J]+2\left[p, \Omega_{h}\right]-\frac{\bar{d}_{i}}{2}\left([p, U]+\left[\phi, \nabla_{\perp}^{2} p\right]\right. \\
\left.+\nabla_{\perp}^{2}[p, \phi]\right)+\nu_{\perp} \nabla_{\perp}^{2}\left\{U+\bar{d}_{i} \nabla_{\perp}^{2}\left(p-p_{e q}\right)\right\} \\
\frac{\partial \psi}{\partial t}=\left[\psi, \phi-\bar{d}_{i} p\right]+\eta\left(J-J_{e q}\right), \\
\frac{\partial p}{\partial t}=[p, \phi]+2 \beta \bar{d}_{i}[\psi, J]+\beta\left[\psi, v_{\|}\right]+2 \beta\left[\Omega_{h}, \phi-\bar{d}_{i} p\right] \\
+\chi \nabla_{\perp}^{2}\left(p-p_{e q}\right), \\
\frac{\partial v_{\|}}{\partial t}=\left[v_{\|}, \phi\right]+[\psi, p]+\nu_{\|} \nabla_{\perp}^{2} v_{\|},
\end{gathered}
$$

respectively, where $\quad J=\nabla_{\perp}^{2} \psi, U=\nabla_{\perp}^{2} \phi,[f, g]=\frac{\partial f}{\partial x} \frac{\partial g}{\partial y}$ $-\frac{\partial f}{\partial y} \frac{\partial g}{\partial x}$, and $\nabla_{\perp}=\hat{\mathbf{e}}_{x} \frac{\partial}{\partial x}+\hat{\mathbf{e}}_{y} \frac{\partial}{\partial y}$. In these equations, $U, \phi, \psi$, $J, p, v_{\|}$, and $\Omega_{h}$ are the vorticity, the electrostatic potential, the magnetic flux, the plasma current, the pressure, the parallel velocity, and the magnetic curvature, respectively. The magnetic field and the $\mathbf{E} \times \mathbf{B}$ velocity are represented by $\mathbf{B}=\hat{\mathbf{e}}_{z}-\hat{\mathbf{e}}_{z} \times \nabla \psi \quad$ and $\quad \mathbf{v}_{\mathbf{E} \times \mathbf{B}}=-\nabla \phi \times \hat{\mathbf{e}}_{z}$, respectively. Here, $\hat{\mathbf{e}}_{x}, \hat{\mathbf{e}}_{y}$, and $\hat{\mathbf{e}}_{z}$ are the unit vectors in the $x, y$, and $z$ directions, respectively. The normalizations are $\left[x / a, y / a, \epsilon v_{a} t / a\right.$, $\left.\psi /\left(\epsilon B_{0} a\right), \phi /\left(\epsilon v_{a} B_{0} a\right), \beta_{e} p\left(\epsilon T_{e}\right), v_{\|} /\left(\epsilon v_{a}\right)\right] \rightarrow\left[x, y, t, \psi, \phi, p, v_{\|}\right]$, where $B_{0}$ is the amplitude of the ambient magnetic field along the $z$-axis, $\epsilon$ is the inverse aspect ratio, and $a$ is the typical length of the system size such as a plasma minor radius. Here, $\epsilon$ is assumed to be $\epsilon=0.6 / 3.6$. The parameters $\bar{d}_{i}=d_{i} /(2 a)=c /\left(2 \omega_{p i} a\right)=\left(2 \omega_{c i} \tau_{a}\right)^{-1}$ and $\beta=\beta_{e} /\left(1+\beta_{e}\right)$ are the normalized ion skin depth and the normalized electron beta value, where $\tau_{a}=a / v_{A}, \omega_{c i}$, and $\beta_{e}$ are the Alfvén time, the ion cyclotron frequency, and the electron beta value.

We briefly describe the algorithm of the simulation code used in this paper. ${ }^{8,12}$ Time advancement is made with a second order predictor-corrector method. The simulation box size is $\left(1, L_{y}\right)$ with $L_{y}=4.4$, and the box is assumed to be periodic in the poloidal direction $y$. The plasma is assumed to be surrounded by the perfect conducing wall at $x=-0.5$ and at 0.5 . The derivatives with respect to $x$ are approximated by a second order central finite differential method. Fourier decomposition is used in the $y$ direction as $\psi=\psi_{e q}+\sum_{n} \tilde{\psi}_{n} e^{2 i \pi n y / L_{y}}, \quad \phi=\sum_{n} \tilde{\phi}_{n} e^{2 i \pi n y / L_{y}}, \quad p=p_{e q}$ $+\sum_{n} \tilde{p}_{n} e^{2 i \pi n y / L_{y}}$, and $v_{\|}=\sum_{n} \tilde{v}_{\|} e^{2 i \pi n y / L_{y}}$, where $n$ is the mode number and the subscript $e q$ represents the equilibrium quantity. Here, we assumed that the equilibrium $\mathbf{E} \times \mathbf{B}$ velocity is zero, i.e., $\phi_{e q}=0$. The number of grid points in the $x$ direction is 1025 , and the total number of the Fourier modes ranges from 40 to 200 depending on the strength of the nonlinear mode coupling.

The equilibrium profiles of the magnetic field and the pressure are set to be

$$
\psi_{e q}=-B_{0} L_{s} \ln \left[\cosh \left(\frac{x}{L_{s}}\right)\right],
$$

$$
p_{e q}=\frac{\beta_{t}}{2 \epsilon}\left\{1-\Delta P_{0} \tanh \left(\frac{x}{L_{p}}\right)\right\}
$$

where $L_{s}=L_{p}=0.15, B_{0}=0.1617, \Delta P_{0}=0.8235$, and $\beta_{t}$ is the total beta value defined as $\beta_{t}=2 \beta_{e}$ assuming that the ion pressure is the same as the electron pressure. In order to study resistive interchange modes, the equilibrium current density gradient is set to be zero, and the magnetic curvature term and the total beta values are chosen to be $\Omega_{h}=1.481 x$ and $\beta_{t}=0.005$. The dissipation coefficients are assumed to be uniform, and their normalized value are chosen as $\eta=\nu_{\perp}=\nu_{\|}=\chi=10^{-6}$.

\section{PARITY}

The linear instabilities described by the two-fluid model, Eqs. (1)-(4), are categorized into two groups. One is the interchange parity and the other is the tearing parity, and they are related to the symmetry in the $x$ direction. When the perturbed electrostatic potential is an even function of $x$, i.e., $\phi(-x, y, t)=\phi(x, y, t)$, the instability is called as the interchange parity mode. On the other hand, when the electrostatic potential is an odd function of $x$, i.e., $\phi(-x, y, t)$ $=-\phi(x, y, t)$, the instability is called as the tearing parity mode. The parity of other quantities is summarized in Table I. The interchange parity and tearing parity modes are independent during their linear growing evolution; however, they can interact each other in their nonlinear evolution. Thus, their nonlinear interactions have a significant impact on their saturation levels, especially on the magnetic island formation as described in Ref. 10 .

In this section, we explain the parity symmetry of the reduced MHD model

$$
\begin{gathered}
\frac{\partial}{\partial t} \nabla^{2} \phi+\left[\phi, \nabla^{2} \phi\right]=\left[\psi, \nabla^{2} \psi\right], \\
\frac{\partial}{\partial t} \psi+[\phi, \psi]=\eta \nabla^{2} \psi,
\end{gathered}
$$

instead of the two-fluid model for simplicity, where $\nabla_{\perp}^{2}$ is replaced by $\nabla^{2}$ in this section. We consider the parity symmetry against $x$ by defining the parity transformation as $^{10,13,14}$

$$
\mathcal{P}_{x} Q(x, y, t)=Q(-x, y, t)
$$

The parity symmetry against $y$ is related to the symmetry along the magnetic field line in a torus plasma. ${ }^{16-18}$

TABLE I. Parity of perturbation under the transformation $\mathcal{P}_{x} Q(x, y)$ $=Q(-x, y) . \hat{\phi}$ is the electrostatic potential perturbation, $\hat{\psi}$ is the magnetic flux perturbation, $\hat{v}_{\|}$is the parallel velocity perturbation, $\hat{J}$ is the parallel current density perturbation, and $\hat{p}$ is the pressure perturbation.

\begin{tabular}{lccccc}
\hline \hline & $\hat{\phi}$ & $\hat{\psi}$ & $\hat{v}_{\|}$ & $\hat{J}$ & $\hat{p}$ \\
\hline Interchange parity mode & Even & Odd & Odd & Odd & Even \\
Tearing parity mode & Odd & Even & Even & Even & Odd \\
\hline \hline
\end{tabular}




\section{A. Parity conservation of linear instability}

In this subsection, we describe that both tearing and interchange parity modes satisfy the linearized reduced MHD equations so that each parity is conserved during the linear evolution. We consider a normal shear plasma and assume that $B_{y}(-x)=-B_{y}(x), \quad \psi_{e q}(-x)=\psi_{e q}(x)$, for instance, $B_{y}=B_{0} x, \psi_{e q}=B_{0} x^{2} / 2$, where $\psi(\mathbf{x}, t)=\psi_{e q}(x)$ $+\hat{\psi}(\mathbf{x}, t), \phi(\mathbf{x}, t)=\hat{\phi}(\mathbf{x}, t)$, and $\mathbf{x}=(x, y)$. We apply the parity transformation $\mathcal{P}_{x}$ to the linearized equation, and then, we have

$$
\begin{gathered}
\frac{\partial}{\partial t} \nabla^{2} \hat{\phi}_{ \pm}=\left[\psi_{e q}, \nabla^{2} \hat{\psi}_{\mp}\right]+\left[\hat{\psi}_{\mp}, \nabla^{2} \psi_{e q}\right] \\
\frac{\partial}{\partial t} \hat{\psi}_{\mp}+\left[\hat{\phi}_{ \pm}, \psi_{e q}\right]=\eta \nabla^{2} \hat{\psi}_{\mp},
\end{gathered}
$$

(Appendix A) by using the fact that the transformation changes the sign of the Poisson bracket $\mathcal{P}_{x}[f, g]$ $=-\left[\mathcal{P}_{x} f, \mathcal{P}_{x} g\right]$. The equation implies that the tearing parity mode $\left(\hat{\phi}_{-}, \hat{\psi}_{+}\right)$and the interchange parity mode $\left(\hat{\phi}_{+}, \hat{\psi}_{-}\right)$ satisfy the linearized equation, respectively, and thus, the parities are conserved during the linear evolution, respectively, where $\hat{\phi}_{ \pm}(x, y, t)=\frac{1}{2}\left(1 \pm \mathcal{P}_{x}\right) \hat{\phi}(x, y, t), \hat{\psi}_{ \pm}(x, y, t)$ $=\frac{1}{2}\left(1 \pm \mathcal{P}_{x}\right) \hat{\psi}(x, y, t)$. In terms of $\phi_{ \pm}$and $\psi_{ \pm}$, the interchange parity is $\mathcal{P}_{x} \hat{\psi}_{-}(x)=\hat{\psi}_{-}(-x)=-\hat{\psi}_{-}(x)$ and $\mathcal{P}_{x} \hat{\phi}_{+}(x)=\hat{\phi}_{+}(-x)=\hat{\phi}_{+}(x)$, and the tearing parity is $\mathcal{P}_{x} \hat{\phi}_{-}(x)=\hat{\phi}_{-}(-x)=-\hat{\phi}_{-}(x)$ and $\mathcal{P}_{x} \hat{\psi}_{+}(x)=\hat{\psi}_{+}(-x)$ $=\hat{\psi}_{+}(x)$.

We have shown that each of the interchange and tearing parities is conserved, when the amplitude of an instability is small and the linearized equation is valid. It is noted that these originate from the parity symmetry of the Poisson bracket, and so, they are valid not only for reduced fluid models but for the gyrokinetic equations.

\section{B. Nonlinear parity mixing}

Nonlinear effects become important and control the saturation amplitude of instability when an instability grows. We will show that the interchange parity mode is not able to get saturated with keeping its parity, and thus, the interchange parity mode does not satisfy the nonlinear equation. The constraint on the parity symmetry controls the nonlinear evolution of the interchange mode. This is true for not only the MHD instability but also the drift-wave instability such as the ion temperature gradient (ITG) mode because the gyrokinetic equation has the same parity symmetry. Hence, the interchange parity mode should produce the tearing parity mode in nonlinear evolution, which is called nonlinear parity mixing. ${ }^{10}$

The nonlinear parity mixing occurs when the interchange parity mode grows and then the tearing parity mode is excited by the interchange parity mode through nonlinear effects. In order to show this, we substitute the electrostatic potential $\phi=\hat{\phi}_{+}+\hat{\phi}_{-}$and the magnetic potential $\psi=\psi_{\text {eq }}$ $+\hat{\psi}_{+}+\hat{\psi}_{-}$to the reduced MHD equations. We neglect the linear terms to elucidate the nonlinear parity mixing as

$$
\begin{aligned}
& \frac{\partial}{\partial t} \nabla^{2} \hat{\phi}_{+}+\frac{\partial}{\partial t} \nabla^{2} \hat{\phi}_{-}=-\left[\hat{\phi}_{+}, \nabla^{2} \hat{\phi}_{+}\right]-\left[\hat{\phi}_{-}, \nabla^{2} \hat{\phi}_{-}\right] \\
&-\left[\hat{\phi}_{+}, \nabla^{2} \hat{\phi}_{-}\right]-\left[\hat{\phi}_{-}, \nabla^{2} \hat{\phi}_{+}\right] \\
&+ {\left[\hat{\psi}_{+}, \nabla^{2} \hat{\psi}_{+}\right]+\left[\hat{\psi}_{-}, \nabla^{2} \hat{\psi}_{-}\right] } \\
&+ {\left[\hat{\psi}_{+}, \nabla^{2} \hat{\psi}_{-}\right]+\left[\hat{\psi}_{-}, \nabla^{2} \hat{\psi}_{+}\right] } \\
& \frac{\partial}{\partial t} \hat{\psi}_{+}+\frac{\partial}{\partial t} \hat{\psi}_{-}=-\left[\hat{\phi}_{+}, \hat{\psi}_{-}\right]-\left[\hat{\phi}_{-}, \hat{\psi}_{+}\right]-\left[\hat{\phi}_{+}, \hat{\psi}_{+}\right] \\
&-\left[\hat{\phi}_{-}, \hat{\psi}_{-}\right] .
\end{aligned}
$$

First, we show that the tearing parity mode does not produce the interchange parity mode even in nonlinear evolution so that the tearing parity mode satisfies the nonlinear equation. The tearing parity mode is represented by $\left(\hat{\phi}_{-}, \hat{\psi}_{+}\right) \neq$ 0 and $\left(\hat{\phi}_{+}, \hat{\psi}_{-}\right)=0$. We substitute them into the right-handside of Eqs. (12) and (13), and then, we have

$$
\begin{gathered}
\frac{\partial}{\partial t} \nabla^{2} \hat{\phi}_{-}=-\left[\hat{\phi}_{-}, \nabla^{2} \hat{\phi}_{-}\right]+\left[\hat{\psi}_{+}, \nabla^{2} \hat{\psi}_{+}\right], \quad \frac{\partial}{\partial t} \nabla^{2} \hat{\phi}_{+}=0 \\
\frac{\partial}{\partial t} \hat{\psi}_{+}=-\left[\hat{\phi}_{-}, \hat{\psi}_{+}\right], \quad \frac{\partial}{\partial t} \hat{\psi}_{-}=0 .
\end{gathered}
$$

Equations (14) and (15) include only the tearing parity mode, and thus, there is no nonlinear parity mixing, when the tearing parity mode grows nonlinearly, e.g., in the Rutherford regime. This means that when the initial state includes only the tearing parity mode, the interchange parity mode is not generated since the rate of the change of the interchange parity mode is zero.

Next, we show the excitation of the tearing parity mode by the interchange parity mode through the nonlinear parity mixing so that the interchange parity mode does not satisfy the nonlinear equation and is not able to get saturated with keeping its parity. We substitute the interchange parity mode $\left(\hat{\phi}_{+}, \hat{\psi}_{-}\right) \neq 0$ and $\left(\hat{\phi}_{-}, \hat{\psi}_{+}\right)=0$ to the right-hand-side of Eqs. (12) and (13), and then, we obtain

$$
\begin{gathered}
\frac{\partial}{\partial t} \nabla^{2} \hat{\phi}_{-}=-\left[\hat{\phi}_{+}, \nabla^{2} \hat{\phi}_{+}\right]+\left[\hat{\psi}_{-}, \nabla^{2} \hat{\psi}_{-}\right], \quad \frac{\partial}{\partial t} \nabla^{2} \hat{\phi}_{+}=0 \\
\frac{\partial}{\partial t} \hat{\psi}_{+}=-\left[\hat{\phi}_{+}, \hat{\psi}_{-}\right], \quad \frac{\partial}{\partial t} \hat{\psi}_{-}=0 .
\end{gathered}
$$

The nonlinear terms $\left[\hat{\phi}_{+}, \nabla^{2} \hat{\phi}_{+}\right],\left[\hat{\psi}_{-}, \nabla^{2} \hat{\psi}_{-}\right],\left[\hat{\phi}_{+}, \hat{\psi}_{-}\right]$in the right-hand-side of Eqs. (16) and (17) represent that the nonlinear interactions of the interchange parity mode excite the tearing parity mode, i.e., the nonlinear parity mixing produces the tearing parity mode. ${ }^{10}$ This results in the coexistence of both parity modes $\left(\hat{\phi}_{+}, \hat{\psi}_{-}\right) \neq 0$ and $\left(\hat{\phi}_{-}, \hat{\psi}_{+}\right) \neq 0$, and then, the tearing parity mode is excited by nonlinear interactions between the same parity modes, while the interchange parity mode is excited by nonlinear interactions between opposite parity modes as shown in Appendix B. 


\section{Modulational parity instability}

One of the nonlinear parity mixing processes is a modulational instability. A saturated state of the interchange mode is unstable against the tearing parity mode, and this instability is called as the modulational parity instability. The modulational instability is normally used to investigate the zonal flow production by a saturated drift-wave instability called a pump mode. ${ }^{15}$ In the modulational parity instability, the pump mode is the interchange mode, and the side-band mode corresponds to the tearing parity mode representing the magnetic island formation. In order to show the modulational instability, we suppose that the interchange mode $\left(\hat{\Phi}_{+}(\mathbf{x}),\left(\hat{\Psi}_{-}(\mathbf{x})\right)\right.$ with $\hat{\Phi}_{-}=\hat{\Psi}_{+}=0$ gets saturated and is in a steady state. Then, we add perturbations to the saturate state of the interchange mode. The perturbations are a tearing parity mode $\left(\hat{\phi}_{-}(\mathbf{x}, t),\left(\hat{\psi}_{+}(\mathbf{x}, t)\right)\right.$ with $\hat{\phi}_{+}=\hat{\psi}_{-}=0$ and an interchange parity zonal flow $\left(\hat{\phi}_{+0}(\mathbf{x}, t),\left(\hat{\psi}_{-0}(\mathbf{x}, t)\right)\right.$ with $\hat{\phi}_{-0}=\hat{\psi}_{+0}=0$. We assume that the perturbations are much smaller than the interchange mode, $\left(\hat{\phi}_{-}, \hat{\psi}_{+}\right) \ll\left(\hat{\Phi}_{+}, \hat{\Psi}_{-}\right)$ and $\left(\hat{\phi}_{+0}, \hat{\psi}_{-0}\right) \ll\left(\hat{\Phi}_{+}, \hat{\Psi}_{-}\right)$. Substituting them into Eqs. (B1), (B2), (B3), and (B4) yields

$$
\begin{aligned}
& \frac{\partial}{\partial t} \nabla^{2} \hat{\phi}_{-}=- {\left[\hat{\Phi}_{+}, \nabla^{2} \hat{\phi}_{+0}\right]-\left[\hat{\phi}_{+0}, \nabla^{2} \hat{\Phi}_{+}\right] } \\
&+ {\left[\hat{\Psi}_{-}, \nabla^{2} \hat{\psi}_{-0}\right]+\left[\hat{\psi}_{-0}, \nabla^{2} \hat{\Psi}_{-}\right], } \\
& \frac{\partial}{\partial t} \hat{\psi}_{+}=-\left[\hat{\Phi}_{+}, \hat{\psi}_{-0}\right]-\left[\hat{\phi}_{+0}, \hat{\Psi}_{-}\right] \\
& \frac{\partial}{\partial t} \nabla^{2} \hat{\phi}_{+0}=-\left[\hat{\Phi}_{+}, \nabla^{2} \hat{\phi}_{-}\right]-\left[\hat{\phi}_{-}, \nabla^{2} \hat{\Phi}_{+}\right] \\
&+\left[\hat{\Psi}_{-}, \nabla^{2} \hat{\psi}_{+}\right]+\left[\hat{\psi}_{+}, \nabla^{2} \hat{\Psi}_{-}\right] \\
& \frac{\partial}{\partial t} \hat{\psi}_{-0}=-\left[\hat{\Phi}_{+}, \hat{\psi}_{+}\right]-\left[\hat{\phi}_{-}, \hat{\Psi}_{-}\right] .
\end{aligned}
$$

The essential mechanism of the modulational parity instability can be understood by neglecting the magnetic perturbation and by retaining only the first term in the right-handside of Eqs. (18) and (20):

$$
\frac{\partial}{\partial t} \nabla^{2} \hat{\phi}_{-}=-\left[\hat{\Phi}_{+}, \nabla^{2} \hat{\phi}_{+0}\right]
$$

$$
\frac{\partial}{\partial t} \nabla^{2} \hat{\phi}_{+0}=-\left[\hat{\Phi}_{+}, \nabla^{2} \hat{\phi}_{-}\right]
$$

Combining these equation gives

$$
\frac{\partial^{2}}{\partial t^{2}} \nabla^{2} \hat{\phi}_{-}=\left[\hat{\Phi}_{+},\left[\hat{\Phi}_{+}, \nabla^{2} \hat{\phi}_{-}\right]\right] .
$$

This equation implies the growth of the tearing parity mode $\hat{\phi}_{\text {_ }}$ with the growth rate approximated to the amplitude of the saturated interchange mode $\hat{\Phi}_{+}$. It is remarked that the interchange parity zonal flow grows simultaneously.

The dispersion relation of the modulational parity instability of the saturated state of the interchange mode can be obtained by substituting $\hat{\Phi}_{+}=\bar{\Phi}_{+} \exp (\gamma t+i \mathbf{p} \cdot \mathbf{x}), \hat{\phi}_{-}=\bar{\phi}_{-}$ $\exp (\gamma t+i \mathbf{k} \cdot \mathbf{x}), \hat{\phi}_{+0}=\bar{\phi}_{+0} \exp (\gamma t+i \mathbf{q} \cdot \mathbf{x}), \hat{\Psi}_{+}=\bar{\Psi}_{+} \exp (\gamma t$ $+i \mathbf{p} \cdot \mathbf{x}), \hat{\psi}_{-}=\bar{\psi}_{-} \exp (\gamma t+i \mathbf{k} \cdot \mathbf{x})$, and $\hat{\psi}_{+0}=\bar{\psi}_{+0} \exp (\gamma t$ $+i \mathbf{q} \cdot \mathbf{x}$ ) into Eqs. (18), (19), (20), and (21), where $\mathbf{p}=\left(p_{x}, p_{y}\right), \mathbf{k}=\left(k_{x},-p_{y}\right), \mathbf{q}=\left(q_{x}, 0\right)$, and $\mathbf{p}+\mathbf{k}+\mathbf{q}=0 . \mathrm{We}$ will show that the modulational parity instability plays a crucial role in the magnetic island formation by the interchange mode in Sec. VB.

\section{LINEAR ANALYSIS}

The equilibrium described in Sec. II is unstable against the resistive interchange mode but stable against tearing modes. Figure 1 shows the eigen-mode structure of the resistive interchange mode obtained from a simulation code in which the linearized equations of Eqs. (1)-(4) are solved. The interchange mode is indeed the interchange parity mode described in Sec. III. Figure 2 shows the dependence of the linear growth rate $\gamma$ and the real mode frequency $\omega_{r}$ of the interchange mode on the mode number. As the magnitude of the ion skin depth, which is the two-fluid effect, becomes larger, higher modes are suppressed and the most unstable mode is shifted from a higher mode to a lower mode because of the increase in $\omega_{*}$. The interchange mode is most unstable at $n=16$ for $\bar{d}_{i}=0.02$, at $n=3$ for $\bar{d}_{i}=0.05$, and at $n=1$ for $\bar{d}_{i}=0.08$. In Fig. 2(b), the real mode frequency is normalized by $\omega_{*}=-\bar{d}_{i} k_{y} \frac{d p_{e q}}{d x}$, which is the electron diamagnetic frequency at the equilibrium where $k_{y}=2 \pi n / L_{y}$. The real frequency corresponds to the propagation velocity of the

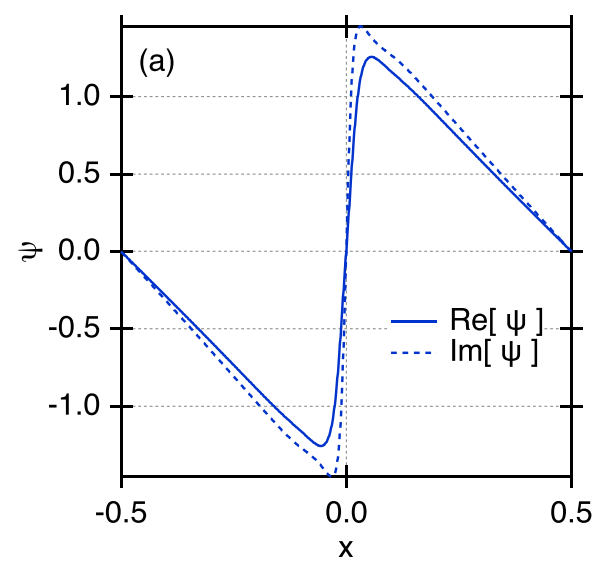

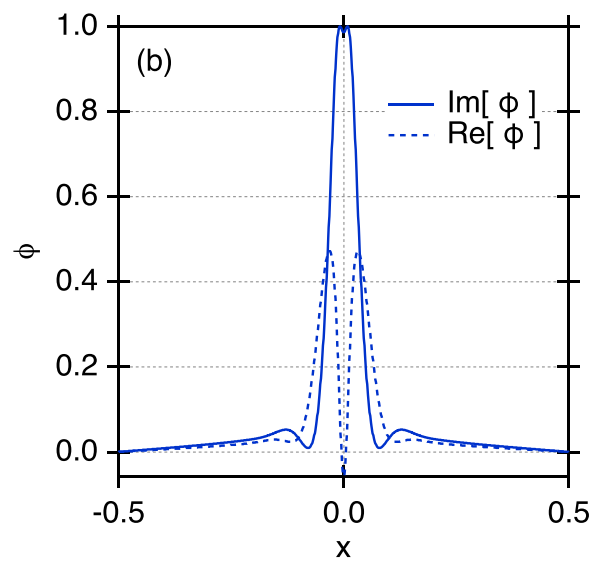

FIG. 1. Eigen-mode structures of (a) the magnetic flux perturbation $\psi$ and (b) the electrostatic potential perturbation $\phi$ of the resistive interchange mode with $n=1$ for $\bar{d}_{i}=0.08$. 

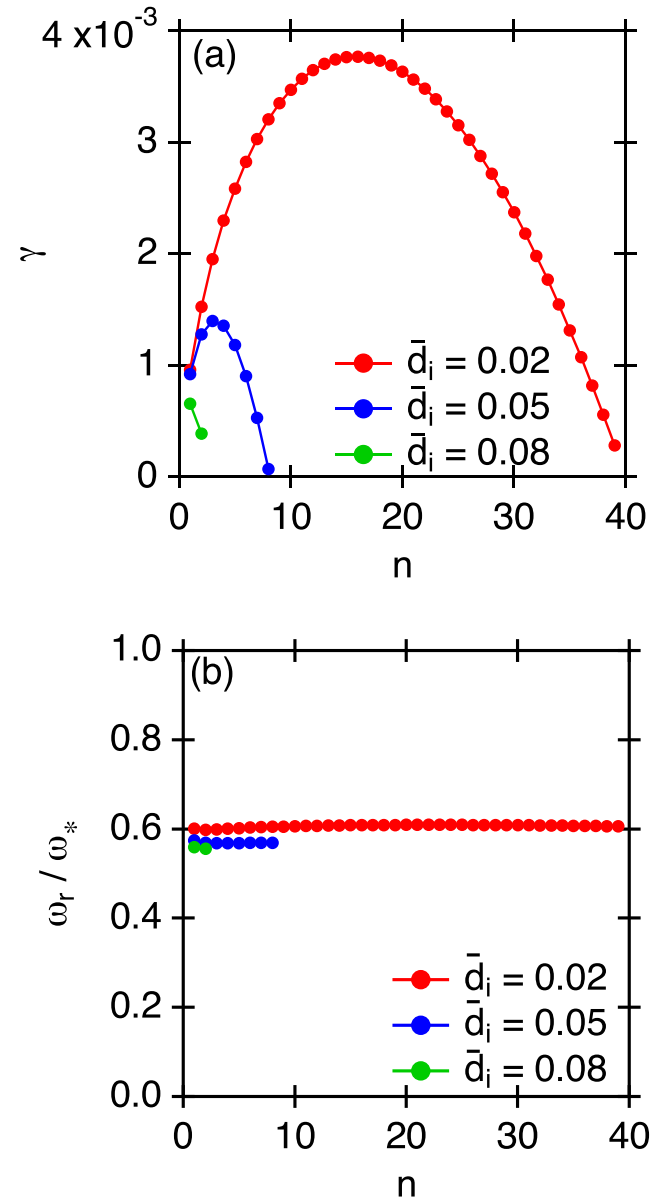

FIG. 2. Dependence of the linear growth rate $\gamma$ and the real mode frequency normalized by the electron diamagnetic frequency $\omega_{r} / \omega_{*}$ on the mode number $n$, which is related to the poloidal wavenumber by $k_{y}=2 \pi n / L_{y}$, for several values of the ion skin depth $\bar{d}_{i}$.

mode in the linear growing evolution. The propagation velocity of the mode is about $60 \%$ of the electron diamagnetic drift velocity $V_{* e, e q}$ regardless of the magnitude of the ion skin depth $d_{i}$, where $V_{* e, e q}=\omega_{*} / k_{y}$. Since there is no equilibrium flow $V_{E \times B, e q}=0$, the electron fluid velocity coincides with the electron diamagnetic drift velocity $V_{e, e q}=V_{* e, e q}+V_{E \times B, e q}=V_{* e, e q}$. Hence, the propagation velocity of the modes is smaller than the electron fluid velocity in the linear phase, which disagrees with the experimental observation of the mode propagation velocity observed in LHD plasmas.

\section{NONLINEAR ANALYSIS ON THE INTERCHANGE MODE PRODUCING MAGNETIC ISLANDS THROUGH PARITY MIXTURES}

The time evolution of the magnetic energy of each Fourier mode for several values of the ion skin depth $\bar{d}_{i}=0.02,0.05$, and 0.08 is shown in Fig. 3, where the magnetic energy for each mode is defined as $E_{M n}=\frac{1}{2} \int_{-0.5}^{0.5} d x$ $\int_{0}^{L_{y}} d y\left|\nabla \tilde{\psi}_{n}\right|^{2}$. The $n=1$ mode becomes the dominant mode in the saturated state for all cases, and the saturated state depends on the ion skin depth $\bar{d}_{i}$. For $\bar{d}_{i}=0.02$, the saturated state is a steady state. On the other hand, the saturated states for $\bar{d}_{i}=0.05$ and $\bar{d}_{i}=0.08$ are not steady states. In this
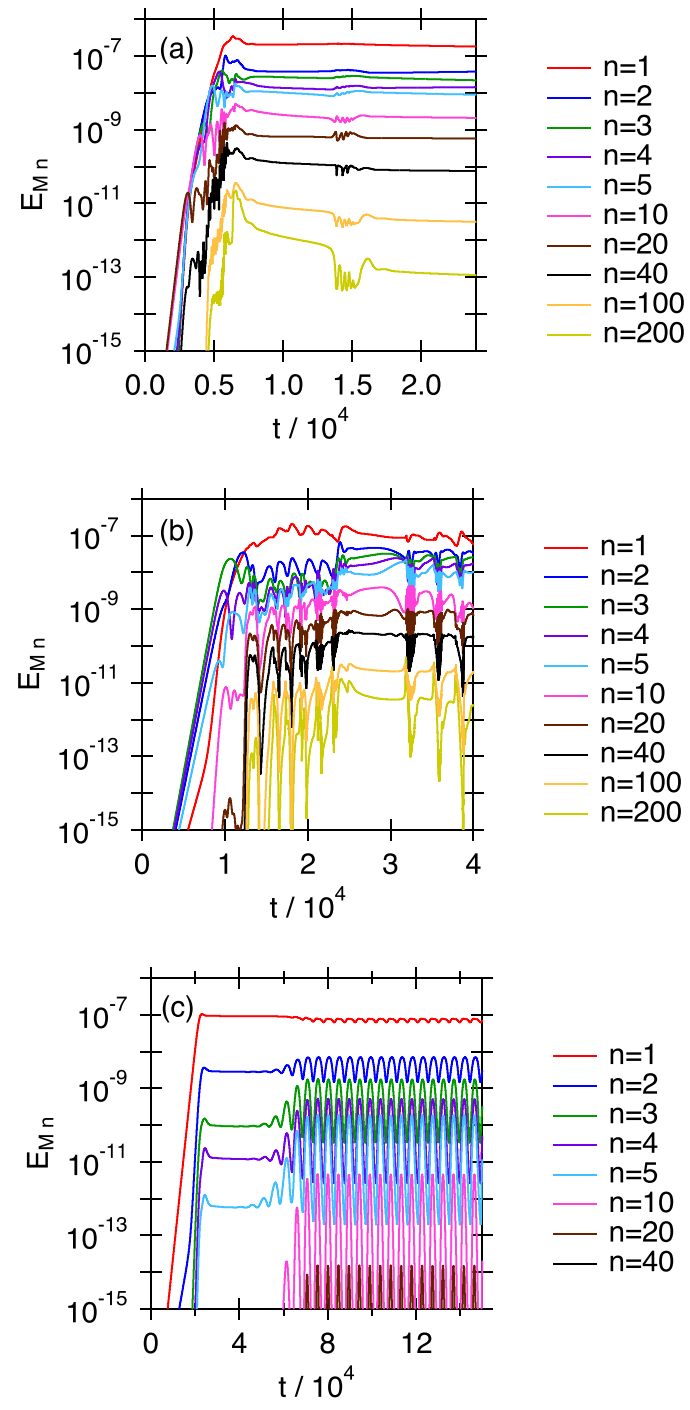

FIG. 3. Time evolution of magnetic energy for each mode $E_{M n}$ for (a) $\bar{d}_{i}=0.02$, (b) $\bar{d}_{i}=0.05$, and (c) $\bar{d}_{i}=0.08$, where $k_{y}=2 \pi n / L_{y}$.

section, the details of the nonlinear evolution for a strongly interchange unstable case with $\bar{d}_{i}=0.02$ and a weakly interchange unstable case with $\bar{d}_{i}=0.08$ are shown. In the former case, the magnetic islands are produced by the parity mixture through nonlinear Fourier mode coupling between two unstable interchange modes. In the latter case, the islands are produced by the parity mixture through the modulational instability.

\section{A. Strongly interchange unstable case}

When the instability is strong with $\bar{d}_{i}=0.02$, there appears a magnetic island in the nonlinear saturated state as shown in Fig. 4, which corresponds to the dominance of the $n=1$ mode. In order to discuss the parity, the variables $\left(\psi, \phi, p, v_{\|}\right)$are decomposed into the even function and the odd function with respect to the neutral magnetic surface, $\left(\psi_{+}+\psi_{-}, \phi_{+}+\phi_{-}, p_{+}+p_{-}, v_{\|+}+v_{\|-}\right)$, where the superscript $+(-)$ denotes the even (odd) function of $x$ as described in Sec. III B. Then, $\left(\psi_{-}, \phi_{+}, p_{+}, v_{\|-}\right)$and $\left(\psi_{+}, \phi_{-}, p_{-}, v_{\|+}\right)$ correspond to the interchange parity and the tearing parity 


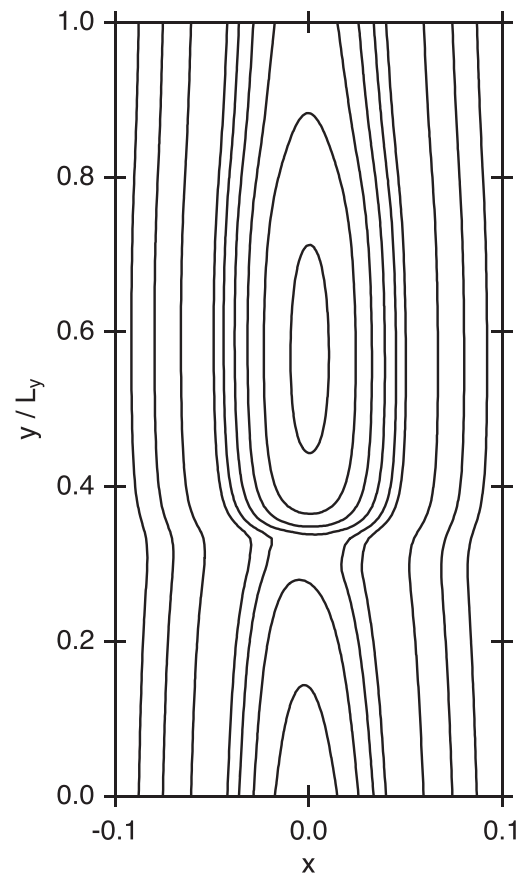

FIG. 4. Magnetic flux surfaces at $t=20000$ for the strongly interchange unstable case with $\bar{d}_{i}=0.02$.

modes, respectively. Figure 5 shows the profiles of the magnetic flux and the electrostatic potential of interchange and tearing parity modes with $n=1$ at $t=20000$. From the linear theory on the parity in Sec. III A, magnetic islands are not formed by the resistive interchange mode during its linear growth since the magnetic flux of the interchange parity mode $\psi_{-}$vanishes at the neutral magnetic surface as shown in Fig. 1. At the nonlinear saturated state, however, the tearing parity mode with finite amplitude at the neutral magnetic surface is generated as shown in Fig. 5(a). This implies that the magnetic island is formed due to the generation of the tearing parity. The amplitudes of tearing parity and interchange parts of the magnetic flux and of the electrostatic potential are comparable. Hence, the mixture of the parities occurs at the saturated state of the interchange mode, and the magnetic islands are formed. The process of the parity mixture, which is the growth of the tearing parity mode with $n=1$, is the nonlinear mode coupling of the linearly unstable interchange modes.
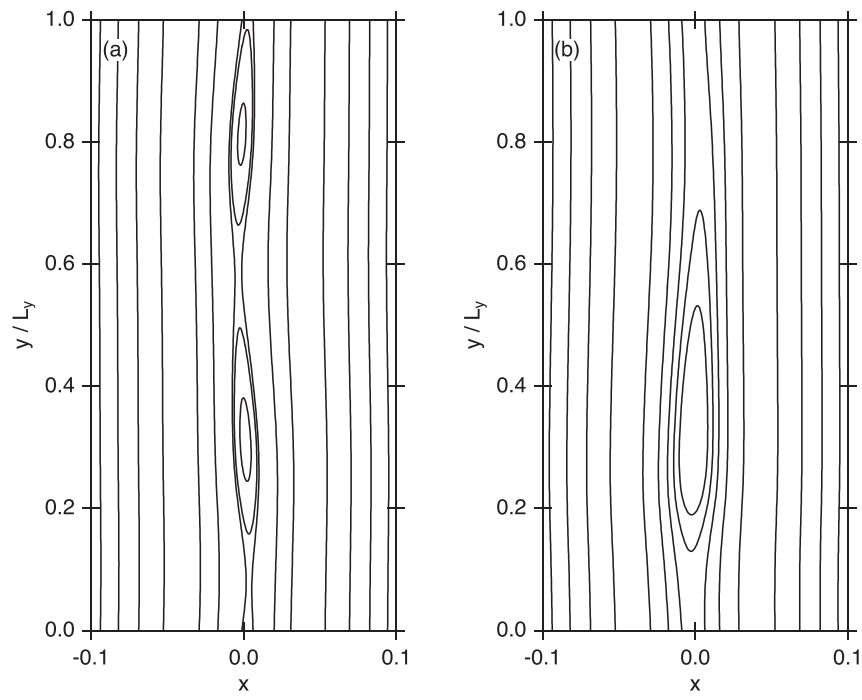

FIG. 6. Magnetic flux surfaces at (a) $t=40000$ in the steady state and (b) $t=150000$ in the oscillatory state for the weakly interchange unstable case $\bar{d}_{i}=0.08$.

\section{B. Weakly interchange unstable case causing modulational parity instability}

When the instability is weak with $\bar{d}_{i}=0.08$, the nonlinear evolution is more complicated than the strongly unstable case with $\bar{d}_{i}=0.02$, and there are two subsequent saturated states as shown in Fig. 3(c). The first subsequent saturated state is a steady state realized after the mode energies become saturated in $20000<t<50000$, and then, the second subsequent saturated state is an oscillatory state at $t>50000$. The numbers of magnetic islands are different in these two states as clearly shown by the magnetic surfaces at $t=40000$ in the steady state and $t=150000$ in the oscillatory state in Fig. 6. Two magnetic islands are formed at $t=40000$ in the steady state, and then, they merge into one wider island at $t=150000$ in the oscillatory state.

First, we examine the steady state with two magnetic islands at $20000<t<50000$ in detail. Figure 7(a) shows the profiles of the tearing parity and the interchange parity parts of the magnetic flux for $n=1$ and $n=2$ at $t=40000$ in the steady state. The amplitude of the tearing parity mode with $n=2$ is larger than that with $n=1$. Thus, two magnetic islands are formed by the tearing parity magnetic flux with $n=2$. This is explained by the mode structure of the
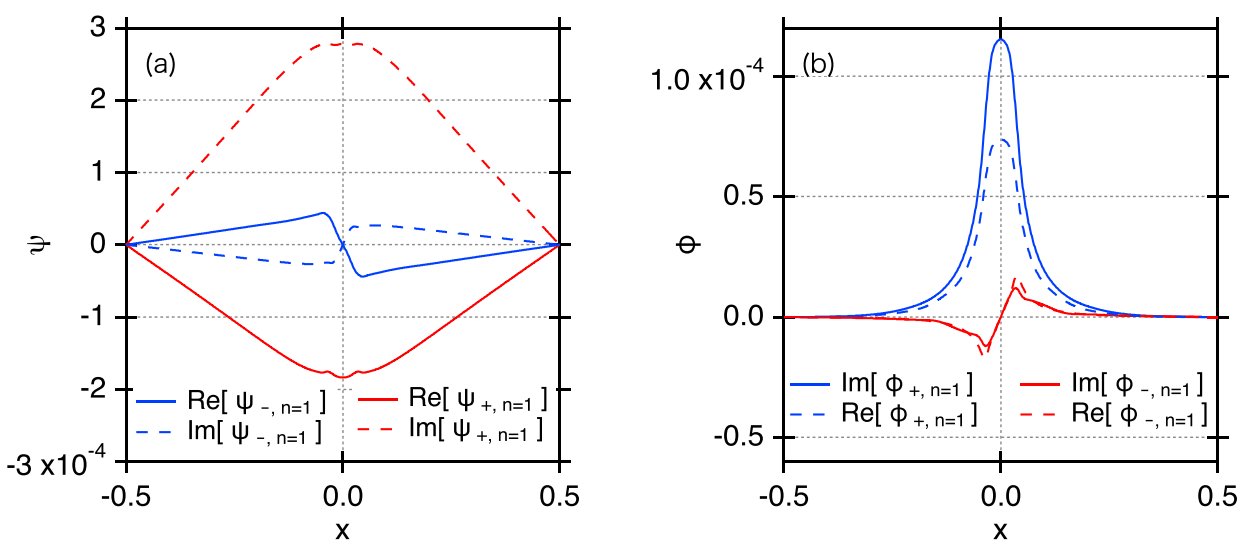

FIG. 5. Profiles of the tearing parity (red curves) and the interchange parity (blue curves) of the $\mathrm{n}=1$ mode for (a) the magnetic flux and (b) the electrostatic potential at $t=20000$ for the strongly interchange unstable case with $\bar{d}_{i}=0.02$. 


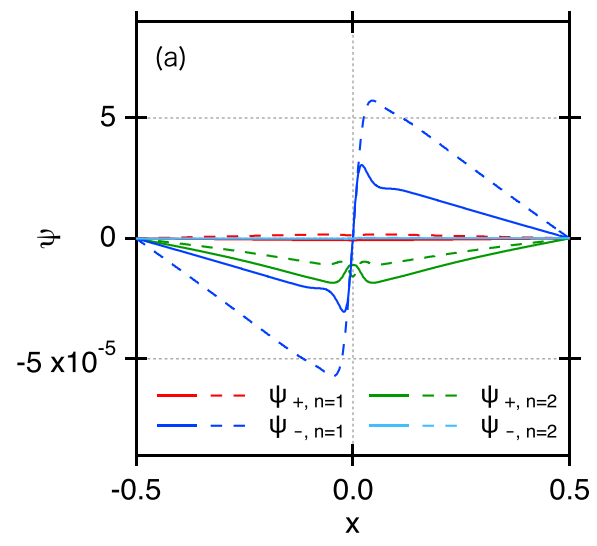

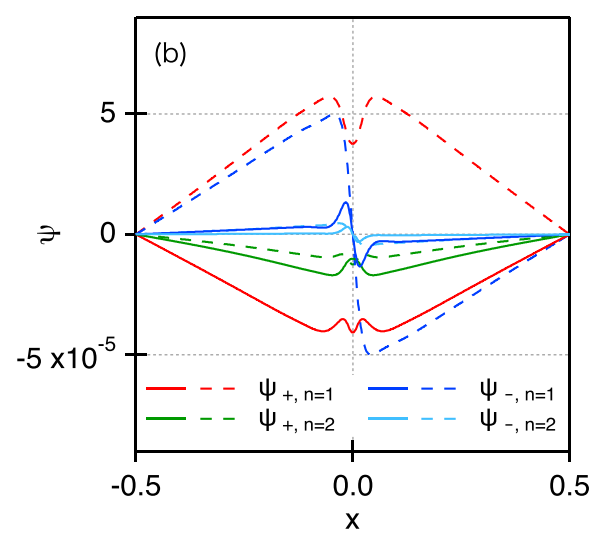

FIG. 7. Profiles of the tearing parity (red and green curves) and the interchange parity (blue and sky blue curves) of the $n=1$ mode and the $n=2$ mode for the magnetic flux at (a) $t=40000$ in the steady state and (b) $t=150000$ in the oscillatory state for the weakly interchange unstable case $\bar{d}_{i}=0.08$. The solid and dashed curves correspond to the real and the imaginary parts, respectively. interchange mode in the poloidal direction. The twisting of the radial displacement with $n=1$ forces magnetic reconnection at two points in the poloidal direction on the neutral magnetic surface so that two X-points are formed and two magnetic islands are produced in Fig. 6(a). Hence, the amplitude of the tearing parity of the $n=2$ mode is larger than that of the $n=1$ mode as shown in Fig. 7(a).

Next, we examine the oscillatory state which has one larger magnetic island shown in Fig. 6 at $t>50000$ in detail. At $t=150000$ in the oscillatory state, the amplitude of the tearing parity part of $n=1$ is larger than that of $n=2$ as shown in Fig. 7(b). The emergence of the tearing parity mode with $n=1$ is caused by the modulational parity instability driven by the saturated $n=1$ interchange mode. Figure 8 shows the time evolution of the kinetic and magnetic energies of the pump mode which is the interchange parity mode with $n=1$ and of the growing side-band modes which are the tearing parity mode with $n=1$ and the asymmetric part of the zonal flow corresponding to the symmetric part of the zonal potential with $n=0$, which has interchange parity. The interchange parity mode with $n=1$ becomes saturated and in the steady state at $t>20000$, and the tearing parity mode with $n=1$ continues to grow with an oscillation during the steady state. Meanwhile, the asymmetric part of the zonal flow grows with the same growth rate as the tearing parity mode with $n=1$, and thus, they are the side-band modes of the modulational instability. Hence, the mechanism of the growth of the tearing parity mode at $t>20000$ is the modulational parity instability driven by the pump mode that is the saturated $n=1$ interchange mode discussed in Sec. III C.

In order to confirm that the modulational parity instability is the cause of the growth of the tearing parity of the $n=1$ mode for $\bar{d}_{i}=0.08$, we set up a similar situation as the one described in Sec. IIIC, i.e., numerical simulations including only the $n=0$ mode and the $n=1$ mode have been carried out by using the simulation data for $\bar{d}_{i}=0.08$. We stop the simulation at $t=30000$ for $\bar{d}_{i}=0.08$ and set the amplitude of the perturbation to be zero for $n>1$. Then, we restart the simulation. Since the pump mode is the interchange parity of the $n=1$ mode, we assume that the amplitude of the interchange parity of the $n=1$ mode does not change in time. This mimics the steady state where the amplitude of the $n=1$ mode does not change at $30000<t<50$ 000. The side-band modes are the tearing parity mode with $n=1$ and the interchange parity mode with $n=0$ in this simulation. As discussed in Sec. III, the growth rate of the modulational instability is proportional to the amplitude of the interchange parity mode with $n=1$. In order to compare the simulations with the theoretical prediction, simulations for several values of an amplification factor of the pump mode $\underset{\sim}{\alpha}$ have been performed, where the pump mode is set to be $\tilde{\psi}_{-, n=1}(t)=\alpha \tilde{\psi}_{-, n=1}\left(t=t_{0}\right) e^{i\left(k_{y} y-\omega_{r}\left(t-t_{0}\right)\right)}$, where $t_{0}=30000, \alpha$ is the amplification factor of the pump mode, and $\omega_{r}$ is the real mode frequency at $t=t_{0}$. It is found that the tearing parity mode with $n=1$ is unstable when $\alpha$ ranges $0.9 \leq \alpha \leq 2.4$. The time evolution of the magnetic
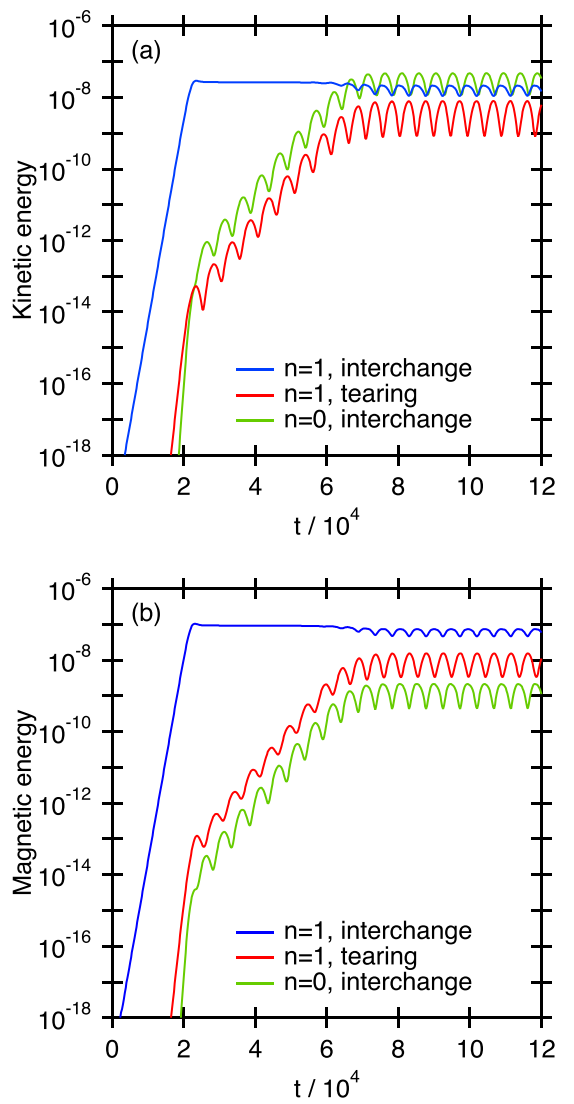

FIG. 8. Time evolution of (a) the kinetic energies and (b) the magnetic energies of both parities of the $n=1$ mode and the interchange parity of the $n=0$ mode for $\bar{d}_{i}=0.08$. 


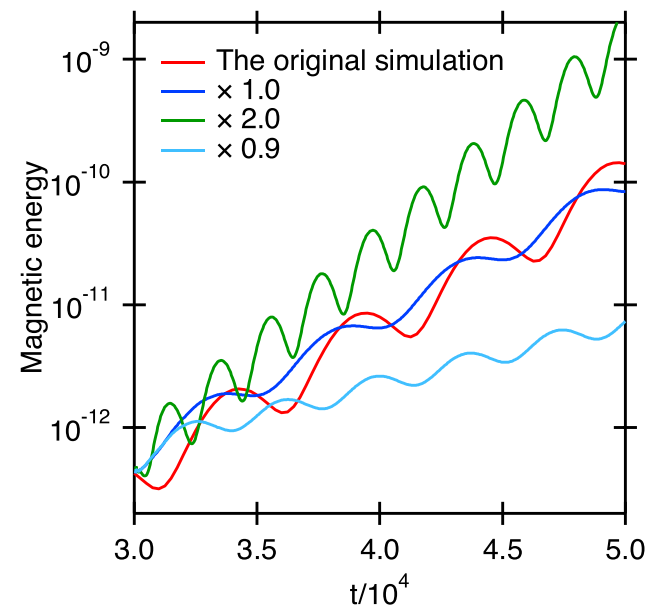

FIG. 9. Time evolution of the magnetic energy of the tearing parity of the $n=1$ mode obtained from the simulations using the $n=0$ mode and the $n=1$ mode. The blue, green, and sky blue curves correspond to the results for $\alpha=1,2$, and 0.9 , respectively. The red curve corresponds to the original simulation result shown in Fig. 8(b).

energy of the tearing parity mode with $n=1$ is shown in Fig. 9 for $\alpha=0.9,1$, and 2. In Fig. 9, the red curve corresponds to the original simulation result shown in Fig. 8(b). The growth rate of the tearing parity of the $n=1$ mode for $\alpha=1$ agrees well with the original simulation result. The slight difference between them may be due to the influence of the $n=2$ mode. The growth rate increases with $\alpha$ for $0.9 \leq \alpha \leq 2$. This implies that the growth of the tearing parity of the $n=1$ mode is due to the modulational parity instability in which the interchange parity mode with $n=1$ is the pump mode.

\section{Propagation velocity of magnetic islands}

The magnetic flux on the neutral magnetic surface becomes finite in the nonlinear phase, and this implies the formation of magnetic islands. The time evolution of the perturbed magnetic flux on the neutral magnetic surface $(x=0)$ for $\bar{d}_{i}=0.02$ is shown in Fig. 10. In the nonlinear phase, since the interchange parity mode with $n=1$ deforms the magnetic surfaces, the X-point and O-point of the magnetic island are not necessarily located at $x=0$. However, the deformation is small as shown in Fig. 4 so that the maximum and minimum of the magnetic flux at each time in Fig. 10 can be regarded as the $\mathrm{O}$-point and the $\mathrm{X}$-point of the

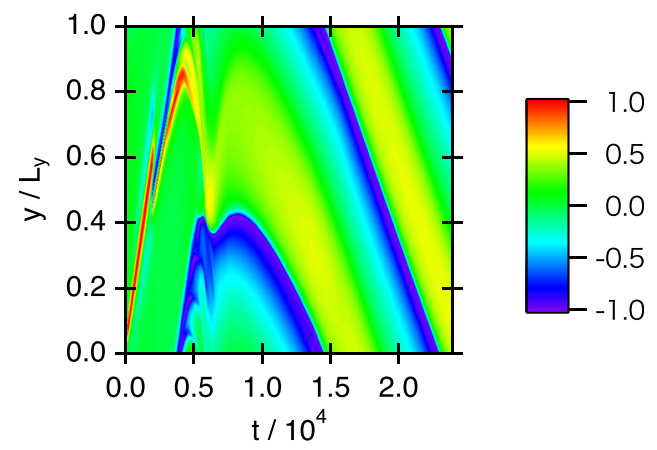

FIG. 10. Time evolution of the perturbed magnetic flux at the neutral magnetic surface for $\bar{d}_{i}=0.02$. The magnetic flux is normalized by the maximum absolute value at each time. magnetic island, respectively. Figure 10 shows the propagation of the mode in the poloidal direction. In the linear phase, the mode propagates in the positive $y$ direction, which corresponds to the equilibrium electron diamagnetic drift velocity direction $V_{* e, e q}$. However, in the nonlinear phase, the propagation direction of the mode is reversed, and the mode propagates in the negative $y$ direction which is the equilibrium ion diamagnetic drift velocity direction $V_{* i, e q}$.

The magnetic island propagation is expected to be controlled by drift velocities, and so, we discuss here the profiles of drift velocities. Figure 11(a) shows the profiles of the zonal flow velocity $V_{E \times B}$, the electron diamagnetic velocity $V_{* e}$, and the electron fluid velocity $V_{e}$ at the saturated state where $V_{E \times B}, V_{* e}$, and $V_{e}$ are defined as $V_{E \times B}=\tilde{\phi}_{n=0}^{\prime}$, $V_{* e}=-\bar{d}_{i}\left(p_{e q}^{\prime}+\tilde{p}_{n=0}^{\prime}\right)$, and $V_{e}=V_{* e}+V_{E \times B}$, respectively. Here, the prime denotes the derivative with respect to $x$. The profile of the electron diamagnetic drift velocity at the initial equilibrium $V_{* e, e q}$ is also shown in Fig. 11(a). Since the pressure profile becomes flattened inside the magnetic island, the electron diamagnetic drift velocity decreases. The zonal flow has both the symmetric (tearing parity) and asymmetric (interchange parity) components with respect to the neutral magnetic surface. Their profiles of the symmetric component are shown in Fig. 11(b). The symmetric component of the
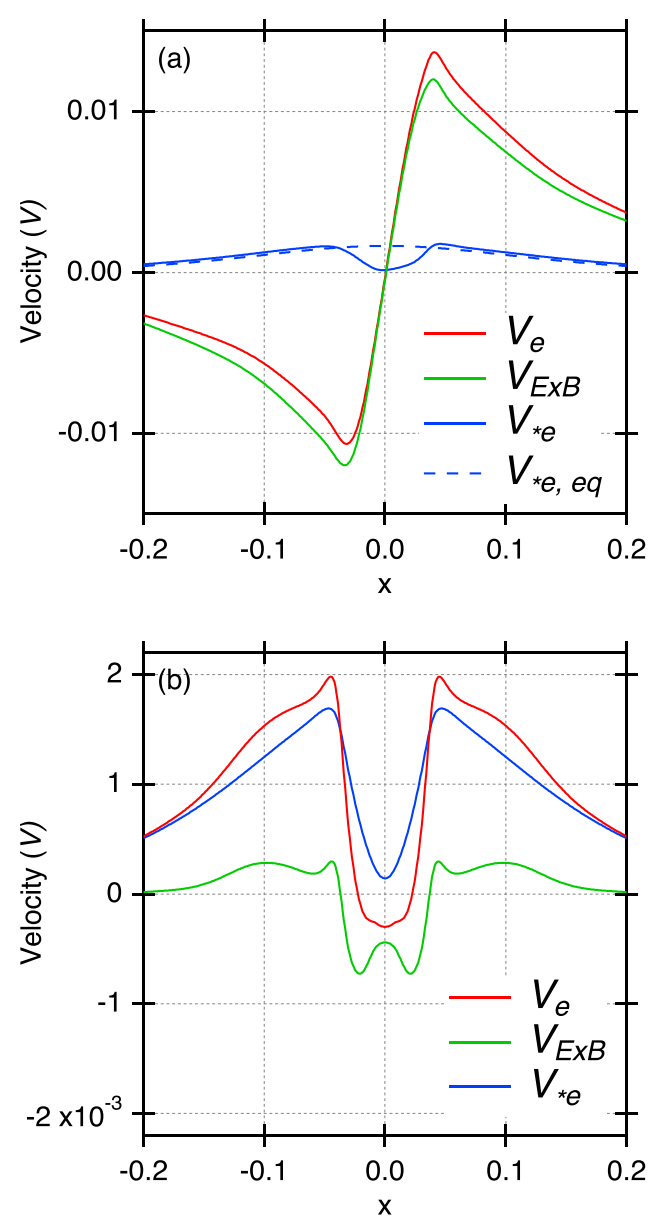

FIG. 11. (a)Profiles of the zonal components of the $\mathbf{E} \times \mathbf{B}$ velocity $\left(V_{E \times B}\right)$, the electron diamagnetic velocity $\left(V_{* e}\right)$, and the electron fluid velocity $\left(V_{e}\right)$ at $t=20000$ for $\bar{d}_{i}=0.02$. The profiles of their symmetric (tearing parity) components are shown in (b). 
zonal flow is generated in the direction of the equilibrium ion diamagnetic drift at the neutral magnetic surface. Since the $\mathbf{E} \times \mathbf{B}$ velocity is larger than the electron diamagnetic drift velocity at the neutral magnetic surface, the direction of the electron fluid is in the equilibrium ion diamagnetic drift direction, which is in the negative $y$ direction and is consistent with the propagation direction of the magnetic island shown in Fig. 10. Figure 12 shows the profiles of the zonal component of the $\mathbf{E} \times \mathbf{B}$ velocity, the electron diamagnetic velocity, and the electron fluid velocity around the neutral sheet at $t=40000$ in the steady state and $t=150000$ in the oscillatory state. At $t=40000$ in the steady state, the profile of the zonal flow is symmetric with respect to the neutral magnetic surface. However, the amplitude of the asymmetric components increases at $t=150000$ in the oscillatory state. This is due to the generation of the interchange parity part of the zonal flow by the modulational parity instability. Figure 13 shows the time evolution of the perturbed magnetic flux at the neutral magnetic surface in the steady state and the oscillatory state. Although the number of the magnetic islands is different, the magnetic islands propagate in the positive $y$ direction, i.e., the equilibrium electron diamagnetic drift direction in both phases. In comparison with $\bar{d}_{i}=0.02$, since the width of the magnetic island for $\bar{d}_{i}=0.08$ is smaller than that for $\bar{d}_{i}=0.02$, the reduction of the pressure gradient
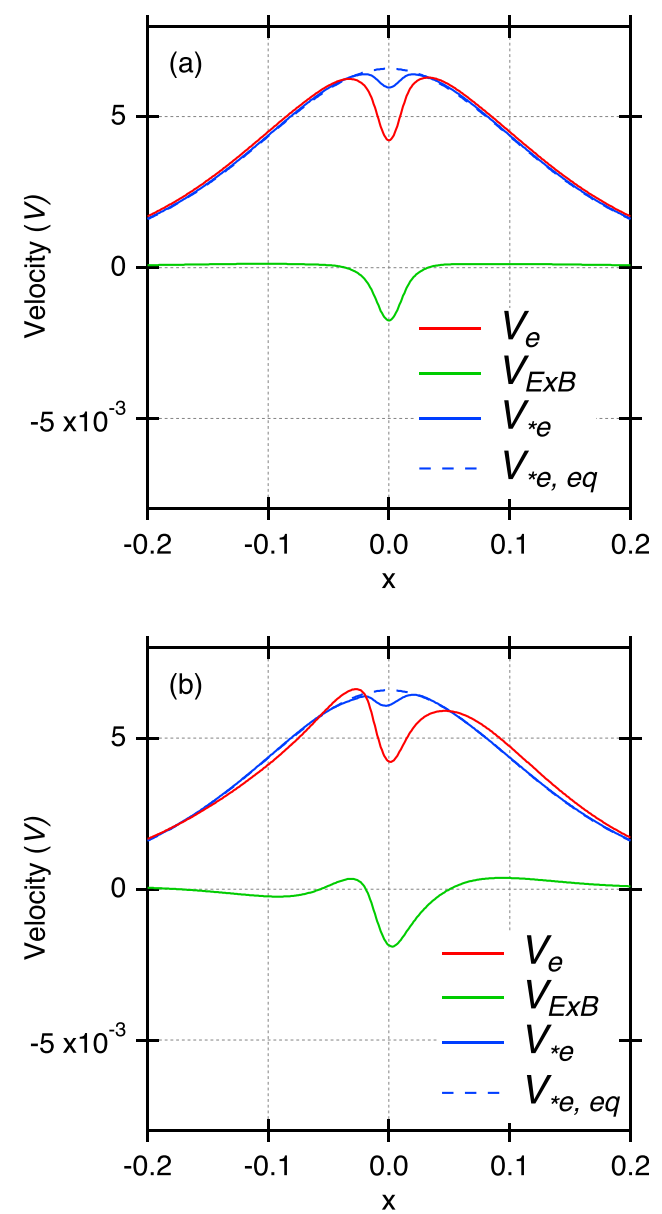

FIG. 12. (a)Profiles of the zonal components of the $\mathbf{E} \times \mathbf{B}$ velocity $\left(V_{E \times B}\right)$, the electron diamagnetic velocity $\left(V_{* e}\right)$, and the electron fluid velocity $\left(V_{e}\right)$ at (a) $t=40000$ and (b) $t=15000$ for $\bar{d}_{i}=0.08$.
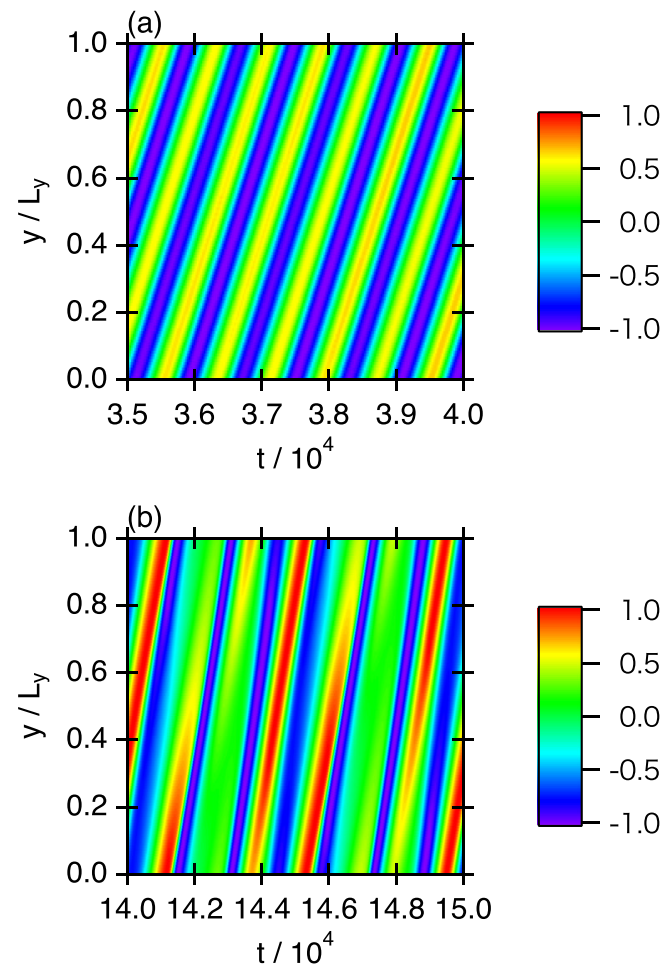

FIG. 13. Time evolution of the perturbed magnetic flux on the neutral magnetic surface $\hat{\psi}(x=0)$ for (a) $35000 \leq t \leq 40000$ and (b) $140000 \leq t$ $\leq 150000$ for $\bar{d}_{i}=0.08$. The magnetic flux is normalized by the maximum absolute value at each time in order to clearly show the $\mathrm{O}$ - and $\mathrm{X}$ - points of the magnetic islands.

for $\bar{d}_{i}=0.08$ is smaller than that for $\bar{d}_{i}=0.02$. Thus, the electron diamagnetic drift velocity is maintained to be larger than the $\mathbf{E} \times \mathbf{B}$ velocity flowing in the ion diamagnetic drift direction at the neutral magnetic surface. As a result, the electron fluid flow is in the equilibrium electron diamagnetic drift direction, which is consistent with the propagation direction of the magnetic islands.

In the oscillatory sate at $t>50000$ for $\bar{d}_{i}=0.08$, the oscillation results from the difference in the propagation velocities of the tearing parity mode with $n=1$, which is generated by the modulational instability, and the interchange parity mode with $n=1$. In this case, the tearing parity mode with $n=1$ propagates in the direction of the electron diamagnetic drift with respect to the interchange parity mode with $n=1$, and the ratio of the propagation velocities of the tearing parity mode with $n=1\left(v_{T}\right)$ to the interchange parity mode with $n=1\left(v_{I}\right)$ is $v_{T} / v_{I} \sim 1.17$. Hence, the $\mathrm{X}$-point and the $\mathrm{O}$-point are not at rest with respect to the interchange parity mode with $n=1$. This results in the oscillation of the $x$ position of the X-point and the O-point. On the other hand, for $\bar{d}_{i}=0.02$, the tearing parity mode with $n=1$ propagates with the same velocity as the interchange parity mode with $n=1$ at the saturated state. Hence, in this case, there is no oscillation of the $x$ position of the $\mathrm{X}$-point and the O-point, and the steady state is achieved.

We summarize the magnetic island propagation velocity and drift velocities. Figure 14 shows the propagation velocity and drift velocities as a function of the magnetic island width at the saturated state. When the width of the magnetic island 


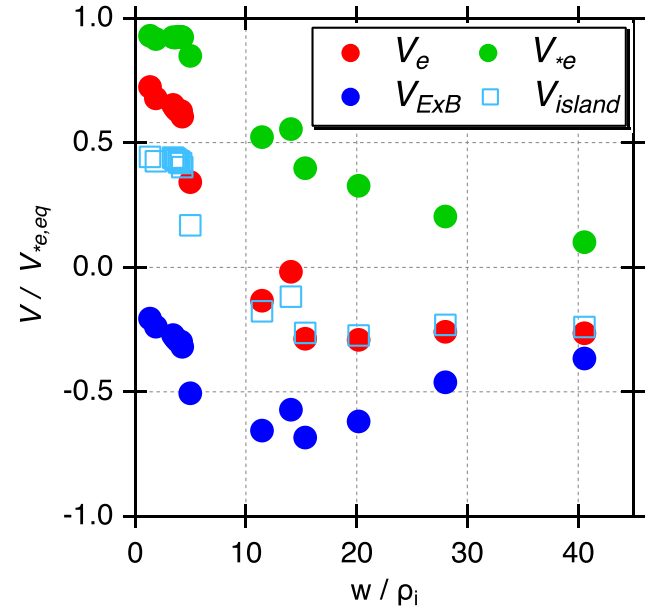

FIG. 14. Dependence of the propagation velocity of the magnetic island ( $\left.V_{\text {island }}\right)$, the electron diamagnetic drift velocity $\left(V_{* e}\right)$, the $\mathbf{E} \times \mathbf{B}$ zonal flow velocity $\left(V_{E \times B}\right)$, and the electron fluid velocity $\left(V_{e}\right)$ on the width of the magnetic island normalized by the ion Larmor radius $\left(\rho_{i}\right)$ at the saturated state.

is about 10 times larger than the ion Larmor radius, the propagation velocity of the magnetic island agrees well with the electron fluid velocity. Thus, the formation of the magnetic island controls the propagation velocity of the resistive interchange modes, which reproduces the velocity of magnetic perturbation of the interchange mode observed in the LHD experiments.

Here, we describe the details of the propagation velocity as a function of the island width, which is controlled by the value of $\bar{d}_{i}$, at the saturated state in Fig. 14. As the width of the magnetic island increases, the pressure profile becomes flattened inside the magnetic island, and thus, the electron diamagnetic drift velocity decreases. The $\mathbf{E} \times \mathbf{B}$ velocity is always in the negative $y$ direction which is the equilibrium ion diamagnetic drift direction. Thus, the direction of the electron fluid velocity depends on the magnitude relationship between the electron diamagnetic drift velocity and the $\mathbf{E} \times \mathbf{B}$ velocity. In conclusion, regardless of the magnitude relation, the propagation velocity of the magnetic island becomes close to the electron fluid velocity as the width of the magnetic island increases. In evaluating the velocities, some averages are used as follows: For $\bar{d}_{i}>0.04$, the saturated state is not a steady state. For such cases, the width of the magnetic island and the propagation velocity are evaluated by averaging over a time period. At the saturated state, the neutral magnetic surface is deformed by the nonlinear effects so that the $x$ position of the neutral magnetic surface depends on $y$. Thus, $V_{E \times B}$ and $V_{* e}$ are evaluated by averaging the velocity on the curved neutral magnetic surface.

\section{SUMMARY AND DISCUSSION}

The propagation velocity of the resistive interchange modes is investigated by nonlinear simulations based on the two-fluid model including both the electron and ion diamagnetic effects. In the linear growing phase, the propagation velocity of the interchange mode is smaller than the electron fluid velocity and is smaller than the observed propagation velocity of the magnetic perturbation of the interchange mode in LHD experiments. On the other hand, due to the nonlinear formation of the magnetic islands, the propagation velocity of the mode becomes close to the electron fluid velocity so that the simulation results reproduce the experimental observation. When the width of the magnetic islands is larger than about 10 times the ion Larmor radius, which can be the observable width of the island in experiments, the propagation velocity of the mode is almost the same as the electron fluid velocity. Therefore, the nonlinear formation of the magnetic island controls the mode rotation velocity of the interchange mode observed in the LHD experiments where the resistive interchange modes rotate with the electron fluid velocity.

We have also shown that the formation of the magnetic islands is due to the generation of the tearing parity mode. The generation of the tearing parity mode occurs in the nonlinear evolution because the pure interchange mode does not satisfy the nonlinear two-fluid equation. This is in contrast to linear evolution, where both parity modes satisfy the linearized two-fluid equation and are conserved. Hence, the energy of the interchange mode should be transferred into the tearing parity mode through nonlinear interactions so that the parity is mixed and the magnetic islands are produced. There are two types of nonlinear parity mixtures between interchange and tearing parity modes. One is the nonlinear interaction between two linearly unstable interchange modes, which is observed when the growth rate of the interchange mode is large with $\bar{d}_{i}=0.02$ and 0.05 . The other is the modulational parity instability observed when the growth of the interchange mode is weak with $\bar{d}_{i}=0.08$. In the former case, the tearing parity mode with $n=1$ grows by the nonlinear mode coupling between the linearly unstable modes, and when the growth of the interchange mode becomes saturated, the $n=1$ interchange mode is the dominant mode and the amplitude of the tearing parity mode with $n=1$ is comparable to the interchange parity of the $n=1$ mode, where $n$ represents the poloidal wavenumber through $k_{y}=2 \pi n / L_{y}$. In the latter case, the nonlinear mode coupling is weak at the beginning and the amplitude of the interchange parity mode with $n=1$ is significantly larger than that of the tearing parity mode in the initial nonlinear saturated state. However, the initial saturated state is unstable against the modulational parity instability. Then, the amplitude of the tearing parity mode with $n=1$ grows and becomes comparable to that of the interchange parity mode. The nonlinear parity mixture explains the reduced MHD simulation results in Refs. 7 and 19 , which show the reduction of the number of the magnetic islands and the $E \times B$ flow generation. It is remarked that the modulational parity instability consists of the growth of the tearing parity mode and the interchange parity zonal flow mode, and these modes are two sides of the same coin. The modulational instability is normally used in the analysis of zonal flow production by the saturated drift-wave instability such as the ITG mode. In the analysis, the drift-wave instability is a pump mode, and a zonal flow mode and a sideband mode are driven by the saturated drift-wave and grow. In our modulational parity instability, the interchange mode is the pump mode, and the tearing parity mode corresponds to the side-band mode. Thus, the analytical method of the 
modulational instability for investigating zonal flow production can be utilized for the analysis of the modulational parity instability.

In the LHD experiments, it is found that the displacement vector of the rotating resistive interchange mode has the interchange parity from the diagnostics of the plasma temperature. $^{2}$ In our simulation results, the saturated state of the resistive interchange mode is the mixture of the interchange parity and the tearing parity. Although the magnetic island is formed due to the tearing parity of the magnetic flux, the amplitude of the interchange parity is significantly larger than that of the tearing parity for the electrostatic potential as shown in Fig. 5(b). Thus, our results are not inconsistent with the experimental results. In other words, the structure of the magnetic field cannot be estimated from the displacement vector or the temperature for the resistive interchange modes. For the locked mode-like instability, the experimental results showed that the non-rotating mode has the tearing parity in the displacement vector. ${ }^{1}$ In this case, the amplitude of the interchange parity may diminish with the suppression of the mode rotation. The study of the mechanism of the suppression of the mode rotation and the mode structure of the locked mode-like instability are future works.

\section{ACKNOWLEDGMENTS}

The authors would like to thank Professors Y. Kishimoto, Y. Nakamura, N. Nakajima, and F. Waelbroeck for helpful discussions. This work was performed on "LHD Numerical Analysis Server" (FUJITSU FX100) of NIFS with the support and under the auspices of the NIFS Collaboration Research program (NIFS16KNXN328).

\section{APPENDIX A: PARITY TRANSFORMATION}

Here, we derive some equations obtained by applying the parity transformation to the nonlinear reduced MHD equations and to the linearized equations. By operating the transformation to the nonlinear equations and using the fact that the transformation changes the sign of the Poisson bracket $\mathcal{P}_{x}[f, g]=-\left[\mathcal{P}_{x} f, \mathcal{P}_{x} g\right]$, we have

$$
\begin{gathered}
\frac{\partial}{\partial t} \nabla^{2}\left(-\mathcal{P}_{x} \phi\right)+\left[\left(-\mathcal{P}_{x} \phi\right), \nabla^{2}\left(-\mathcal{P}_{x} \phi\right)\right]=\left[\mathcal{P}_{x} \psi, \nabla^{2} \mathcal{P}_{x} \psi\right] \\
\frac{\partial}{\partial t} \mathcal{P}_{x} \psi+\left[\left(-\mathcal{P}_{x} \phi\right), \mathcal{P}_{x} \psi\right]=\eta \nabla^{2} \mathcal{P}_{x} \psi
\end{gathered}
$$

Thus, when $(\phi, \psi)$ satisfy the reduced MHD equation, and then, $\left(-\mathcal{P}_{x} \phi, \mathcal{P}_{x} \psi\right)$ also satisfy the equation. This is related to the fact that the tearing parity mode satisfies the nonlinear equation and is able to get saturated in the nonlinear evolution with keeping its parity. When we apply the parity transformation $\mathcal{P}_{x}$ to the linearized equation, we have

$$
\left(\begin{array}{cc}
\frac{\partial}{\partial t} \nabla^{2} & -\left[\psi_{e q}, \nabla^{2}\right]-\left[, \nabla^{2} \psi_{e q}\right] \\
+\left[, \psi_{e q}\right] & \frac{\partial}{\partial t}-\eta \nabla^{2}
\end{array}\right)\left(\begin{array}{c}
\mp \mathcal{P}_{x} \hat{\phi} \\
\pm \mathcal{P}_{x} \hat{\psi}
\end{array}\right)=0 .
$$

The sum and difference of these two equations yield Eqs. (10) and (11).

\section{APPENDIX B: NONLINEAR INTERACTIONS IN COEXISTENCE OF TEARING PARITY AND INTERCHANGE PARITY MODES}

When the tearing parity mode $\left(\hat{\phi}_{-}, \hat{\psi}_{+}\right) \neq 0$ is excited by nonlinear interactions of the interchange parity mode $\left(\hat{\phi}_{+}, \hat{\psi}_{-}\right) \neq 0$, the tearing parity mode is further excited because it obeys

$$
\begin{aligned}
\frac{\partial}{\partial t} \nabla^{2} \hat{\phi}_{-}= & -\left[\hat{\phi}_{+}, \nabla^{2} \hat{\phi}_{+}\right]-\left[\hat{\phi}_{-}, \nabla^{2} \hat{\phi}_{-}\right] \\
+ & {\left[\hat{\psi}_{+}, \nabla^{2} \hat{\psi}_{+}\right]+\left[\hat{\psi}_{-}, \nabla^{2} \hat{\psi}_{-}\right], } \\
\frac{\partial}{\partial t} \hat{\psi}_{+} & =-\left[\hat{\phi}_{+}, \hat{\psi}_{-}\right]-\left[\hat{\phi}_{-}, \hat{\psi}_{+}\right] .
\end{aligned}
$$

The nonlinear terms $\left[\hat{\phi}_{-}, \nabla^{2} \hat{\phi}_{-}\right],\left[\hat{\psi}_{+}, \nabla^{2} \hat{\psi}_{+}\right],\left[\hat{\phi}_{-}, \hat{\psi}_{+}\right]$in the right-hand-side imply that once the tearing parity mode is excited, these nonlinear terms further excite the tearing parity mode. In addition, when both parity modes exist, the interchange parity mode is also produced by nonlinear interactions as

$$
\begin{aligned}
\frac{\partial}{\partial t} \nabla^{2} \hat{\phi}_{+}= & -\left[\hat{\phi}_{-}, \nabla^{2} \hat{\phi}_{+}\right]-\left[\hat{\phi}_{+}, \nabla^{2} \hat{\phi}_{-}\right] \\
+ & {\left[\hat{\psi}_{+}, \nabla^{2} \hat{\psi}_{-}\right]+\left[\hat{\psi}_{-}, \nabla^{2} \hat{\psi}_{+}\right], } \\
\frac{\partial}{\partial t} \hat{\psi}_{-} & =-\left[\hat{\phi}_{-}, \hat{\psi}_{-}\right]-\left[\hat{\phi}_{+}, \hat{\psi}_{+}\right] .
\end{aligned}
$$

The conclusion on the nonlinear interaction in the coexistence of both parity modes is that the tearing parity mode is produced by interactions between the same parity modes, while the interchange parity mode is produced by interactions between opposite parity modes.

\footnotetext{
${ }^{1}$ S. Sakakibara, K. Y. Watanabe, S. Ohdachi, Y. Narushima, K. Toi, K. Tanaka, K. Narihara, K. Ida, T. Tokuzawa, K. Kawahata, H. Yamada, A. Komori, and LHD Experiment Group, Fusion Sci. Technol. 58, 176 (2010).

${ }^{2}$ Y. Takemura, S. Sakakibara, Y. Narushima, M. Okamoto, K. Y. Watanabe, Y. Suzuki, S. Ohdachi, K. Ida, M. Yoshinuma, K. Tanaka, T. Tokuzawa, K. Narihara, I. Yamada, H. Yamada, and LHD Experiment Group, Nucl. Fusion 52, 102001 (2012).

${ }^{3}$ S. Sakakibara, K. Y. Watanabe, H. Yamada, Y. Narushima, T. Yamaguchi, K. Toi, S. Ohdachi, K. Tanak, T. Tokuzawa, K. Narihara, K. Kawahata, A. Komori, and LHD Experimental Group, Plasma Fusion Res. 1, 049 (2006).

${ }^{4}$ Y. Takemura, S. Sakakibara, K. Y. Watanabe, K. Ichiguchi, K. Ida, Y. Suzuki, S. Ohdachi, Y. Narushima, I. Yamada, K. Tanak, H. Yamada, and LHD Experiment Group, Plasma Fusion Res. 8, 1402123 (2013).

${ }^{5}$ M. Wakatani, K. Watanabe, H. Sugama, and A. Hasegawa, Phys. Fluids B 4, 1754 (1992).

${ }^{6}$ T. Nicolas and K. Ichiguchi, Nucl. Fusion 56, 026008 (2016).

${ }^{7}$ K. Ichiguchi and B. A. Carreras, J. Plasma Fusion Res. Ser. 6, 589 (2004).

${ }^{8}$ A. Ishizawa and N. Nakajima, Phys. Plasmas 17, 072308 (2010).

${ }^{9}$ F. L. Waelbroeck, Nucl. Fusion 49, 104025 (2009).

${ }^{10}$ A. Ishizawa and N. Nakajima, see http://www-naweb.iaea.org/napc/physics/ fec/fec2008/papers/th_p8-16.pdf for Proceedings of the 22nd IAEA Fusion Energy Conference, TH/P8-16 (2008).

${ }^{11}$ R. D. Hazeltine, M. Kotschenreuther, and P. J. Morrison, Phys. Fluids 28 2466 (1985).
} 
${ }^{12}$ A. Ishizawa and N. Nakajima, Phys. Plasmas 14, 040702 (2007).

${ }^{13}$ A. Ishizawa, J. Plasma Fusion Res. 92, 530 (2016).

${ }^{14}$ Y. Kishimoto and J. Li, J. Plasma Fusion Res. 92, 552 (2016).

${ }^{15}$ J. Li and Y. Kishimoto, Phys. Plasmas 9, 1241 (2002).

${ }^{16} \mathrm{M}$. Wakatani, Stellarator and Heliotron Devices (Oxford University Press, 1998), Sec. 3.8, p. 99.
${ }^{17}$ H. Sugama, T.-H. Watanabe, M. Nunami, and S. Nishimura, Plasma Phys. Controlled Fusion 53, 024004 (2011).

${ }^{18}$ A. Ishizawa, S. Maeyama, T.-H. Watanabe, H. Sugama, and N. Nakajima, J. Plasma Phys. 81, 435810203 (2015).

${ }^{19}$ K. Ichiguchi, N. Nakajima, and B. A. Carreras, Fusion Sci. Technol. 46, 34 (2004). 